\title{
La politisation des corporations et les révolutions municipales : le cas de Marseille en 1789
}

The Politicization of the Guilds and the Municipal Revolutions: the case of Marseille in 1789

\section{Laurent Henry}

\section{(2) OpenEdition}

\section{Journals}

Édition électronique

URL : https://journals.openedition.org/ahrf/12495

DOI : 10.4000 /ahrf. 12495

ISSN : 1952-403X

Éditeur :

Armand Colin, Société des études robespierristes

Édition imprimée

Date de publication : 1 décembre 2012

Pagination : 27-56

ISBN : 978-2-200-92762-2

ISSN : 0003-4436

Référence électronique

Laurent Henry, « La politisation des corporations et les révolutions municipales : le cas de Marseille en 1789 ", Annales historiques de la Révolution française [En ligne], 370 | octobre-décembre 2012, mis en ligne le 01 décembre 2015, consulté le 23 avril 2022. URL : http://journals.openedition.org/ahrf/12495 ; DOI : https://doi.org/10.4000/ahrf.12495 


\title{
LA POLITISATION DES CORPORATIONS ET LES RÉVOLUTIONS MUNICIPALES : LE CAS DE MARSEILLE EN 1789
}

Laurent HENRY

\begin{abstract}
Les maîtres de corporation ont été des acteurs essentiels du processus révolutionnaire à Marseille en 1789. Avec leurs cahiers de doléances comme programmes politiques, ils furent les artisans d'une première révolution municipale, conquise en deux temps, contre les tenants de l'ordre établi. La prise en compte de l'action des corporations est nécessaire pour comprendre les événements marseillais et mesurer l'autonomie du processus révolutionnaire, par exemple par rapport aux événements parisiens. Les maîtres avaient l'avantage, contrairement aux ouvriers, de disposer de moyens d'actions politiques légaux. Cet épisode est un terrain exceptionnel pour saisir la culture des maîtres en matière d'organisation politique, composante de la culture politique de l'Ancien Régime, dont la connaissance est nécessaire pour comprendre et interpréter l'action des révolutionnaires qui en étaient porteurs.
\end{abstract}

Mots-clés : révolution municipale, assemblée, culture politique, élection, autonomie

Les corporations ont-elles participé au processus révolutionnaire? Si oui, quel sens donner à cette participation? Le point de vue dominant est qu'étant des structures archaïques de production économique, politiquement oligarchiques et qui plus est en déclin, les corporations n'avaient aucune raison de participer au grand mouvement libérateur, politique, que 
fut la Révolution : «marginales par rapport à tout processus social, [les corporations] seront absentes du mouvement pré-révolutionnaire» ${ }^{1}$. Pourtant, même à Paris où les corporations avaient été affaiblies par les réformes de Turgot et Necker en 1776, elles furent actives en 1789, luttant contre leur éviction du processus électoral pour la convocation des États généraux ${ }^{2}$. Et Paris est une exception, car dans le reste du royaume elles n'avaient pas été évincées et la possibilité d'un engagement politique dans une direction proprement révolutionnaire restait ouverte.

Par ailleurs, les réformes de 1776 ne s'appliquèrent pas partout, notamment en Provence. Dans ces régions, le processus révolutionnaire a bien dû entrer en interaction avec un monde corporatif intact. Mais les corporations ont mauvaise réputation chez les historiens. La littérature les concernant a eu tendance à céder à la tentation téléologique de la thèse d'un déclin au XVIII ${ }^{\mathrm{e}}$ siècle ${ }^{3}$. Une batterie d'arguments a été mobilisée pour étayer cette thèse de la " décadence » : l'exaction fiscale de la royauté, la mise sous la tutelle des lieutenants généraux de police, des inspecteurs des manufactures, les entorses internes aux statuts corporatifs, les conflits entre corporations, le refus de l'évolution technique, les critiques formulées par les libéraux. Du point de vue de ces derniers, les corporations sont évidemment en déclin. Mais tous ces arguments ne renseignent en rien sur l'évolution de l'état interne des corporations, et particulièrement sur le degré de légitimité de l'organisation du point de vue de ses membres. Ces arguments signifient plutôt qu'une lutte est engagée contre le modèle corporatif, que l'État royal n'hésite pas à piller les corporations, et qu'il existe une opposition entre au moins deux manières d'organiser la production économique. Par exemple, le fait qu'il y ait des conflits entre corporations ne traduit aucun « déclin ». Au contraire, les conflits pour la délimitation du périmètre productif de chaque corporation sont consubstantiels à la forme corporée d'Ancien Régime, et le fait que des maîtres soient prêts à passer de leur temps et de leur argent pour défendre

(1) Haim BuRstin, Le faubourg Saint-Marcel à l'époque révolutionnaire, Paris, Société des études robespierristes, 1983, p. 96.

(2) Sur la participation des corporations au début du processus révolutionnaire à Paris et en Province, voir Steven L. KAPLAN, La fin des corporations, Paris, Fayard, 2001, p. 363-421.

(3) Étienne MARTIN SAINT-LÉON, Histoire des corporations de métiers, depuis leurs origines jusqu'à leur suppression en 1791, Genève, Slatkine - Megariotis reprints, 1976 (première édition : Paris, 1922) ; Émile COORNAERT, Les corporations en France avant 1789, Paris, Gallimard, 6 édition, 1941 ; Marie-Françoise BENECH-HOCHDOERFFER, « Le déclin des corporations toulousaines », AHRF, 1971, p. 197-220. Voir la très bonne mise au point historiographique dans Bernard GALLINATO, Les corporations à Bordeaux à la fin de l'Ancien Régime. Vie et mort d'un mode d'organisation du travail, Bordeaux, Presses universitaires de Bordeaux, 1992. 
leur activité dans le cadre de la corporation, attesterait plutôt de sa légitimité, aucunement d'une décadence. En fait, aucun critère objectif, évalué par un dépouillement sériel des sources n'étaye la thèse du déclin ${ }^{4}$.

Outre l'imaginaire du déclin qui induit de fausses idées et bloque la production d'hypothèses, il est incorrect d'extrapoler le cas parisien à l'ensemble du royaume ${ }^{5}$. L'examen du cas marseillais montrera notamment que l'organisation politique des communautés de métier diffère substantiellement de celle observée à Paris.

L'hypothèse initiale de ce travail est que la corporation, en tant que forme d'organisation politique, est un lieu favorable à une politisation sur des enjeux de toute nature ${ }^{6}$. Par conséquent les corporations étaient susceptibles d'entrer dans la lutte politique au début de la Révolution, sur les enjeux locaux et nationaux autres que les enjeux strictement corporatifs. La forme corporative est bien une forme d'organisation politique puisqu'elle donne la capacité aux maîtres qui en font partie de participer à des assemblées pour débattre des affaires de la communauté et prendre des décisions les concernant par des votes : «bien plus que le tout-venant des Français et des Françaises, les membres des corporations étaient familiarisés avec les pratiques de la vie politique $»^{7}$. Dans le même ordre d'idée, Philippe Minard a récemment insisté sur le fait que la corporation était « une structure d'auto-organisation [qui] comme telle, ne préjuge en rien du contenu ni du sens des décisions prises $»^{8}$.

Cette culture politique des maîtres, constituée par la maîtrise pratique de procédures visant à résoudre la question du pouvoir dans une communauté de travailleurs, doit retenir l'attention de l'historien de la Révolution, puisque c'est avec cette culture qu'une fraction du peuple urbain rentre en révolution : les révolutionnaires ne sont pas nés en $1789^{9}$.

(4) Il serait pourtant possible de construire des critères mesurant la légitimité du modèle corporatif du point de vue de ses membres, par exemple l'évolution du taux de participation aux assemblées, l'évolution du nombre de demandes de statuts ou du taux de recouvrement des cotes levées annuellement pour les besoins du corps.

(5) Sur l'organisation politique des corporations parisiennes, voir Steven L. KAPLAN, Jeannine ROUTIER PUCCI, Philippe MINARD, «Idéologie, conflits et pratiques politiques dans les corporations parisiennes au XVIII ${ }^{\mathrm{e}}$ siècle », Revue d'histoire moderne et contemporaine, 2002 (1), p. 5-55.

(6) Politisation au sens de prise de position sur des enjeux non purement corporatifs.

(7) Steven L. KAPLAN, La fin..., op. cit., p. 392.

(8) Steven L. KAPLAN, Philippe MiNARD (dir.), La France, malade du corporatisme? $X$ III $^{e}-\mathrm{XX}^{\mathrm{e}}$ siècles, Paris, Belin, 2004, p. 49.

(9) Voir Haim BURSTIN, «Pour une histoire socio-politique du Paris révolutionnaire : réflexions méthodologiques », $A H R F, \mathrm{n}^{\circ} 263,1986, \mathrm{p}$. 22-23, où il met en garde contre la tendance chez les historiens de la Révolution d'un défaut de pratique des archives d'Ancien Régime, qui pourrait les conduire à sous-estimer ou méconnaître certaines de ses institutions, dont font partie les communautés de métiers. 
Il est donc nécessaire de connaître et de caractériser cet ensemble de pratiques, ce dont ce travail rendra compte. La culture d'assemblée dont étaient porteurs les maîtres n'a pu que grandement faciliter l'appropriation des formes politiques mises en place par l'Assemblée nationale, ainsi que leur subversion.

Ce qui doit aussi retenir l'attention, sur le plan événementiel, c'est l'implication des corporations dans la lutte politique tout au long de la période qui va de 1788 à la mise en place des municipalités révolutionnées en février 1790. Si les sources semblent rares à Paris, elles existent en province pour traiter cette question, dans les fonds départementaux et municipaux, respectivement dans les séries E et HH. Dans ces archives, les procès verbaux des assemblées de corporations constituent la source centrale à exploiter. On les complètera si nécessaire et selon les cas par les archives notariales, comme dans le présent travail.

Venons-en à Marseille, centre de cette étude. C'était une ville florissante économiquement à la fin de l'Ancien Régime, peuplée d'environ 120000 âmes $^{10}$. Marseille jouissait encore de quelques privilèges visà-vis du roi, qui par exemple ne pouvait pas y faire entrer ses troupes. Elle avait en Provence le statut de Terre Adjacente et ne participait pas aux États de la Province. Elle resta globalement à l'écart des débats et de la politisation relative à la tenue des États de Provence à Aix. Par ailleurs, c'est à Aix que siégeaient les grandes institutions administratives et judiciaires de la province, comme le Parlement, la Cour des comptes, aides et finances, et l'intendant. L'administration de la ville était confiée à une oligarchie cooptée, coiffée par un maire, un assesseur, quatre échevins, recrutés principalement parmi les négociants. Les charges de lieutenants généraux de police avaient été achetées par la municipalité et les échevins les exerçaient.

L'histoire de Marseille au début de la Révolution souffre d'un certain vide historiographique. En effet, sur la convocation des États généraux et les événements politiques en 1789, l'étude de référence reste celle de C. Lourde, en 1839, qui repose sur des sources locales ${ }^{11}$. Jules Viguier a utilisé en 1895 des sources conservées aux Archives Nationales ${ }^{12}$. Les études ultérieures ont principalement puisé dans Lourde, en commettant

(10) Édouard BARATIER (dir.), Histoire de Marseille, Toulouse, Privat, 1973.

(11) C. LOURDE, Histoire de la Révolution à Marseille et en Provence depuis 1789 jusqu'au Consulat, Marseille, Serres, 1838-1839.

(12) Jules ViguiER, Les débuts de la révolution en Provence, Paris / Marseille, Lenoir / Aubertin, 1895. 
des erreurs factuelles, ne renouvelant pas la connaissance des faits ${ }^{13}$. Il faut attendre les travaux de Monique Cubells, mais qui ne concernent pour Marseille que le printemps 1789, pour voir une reprise des sources, avec de nouveaux éléments factuels versés au dossier, ainsi que la belle édition du journal de Jean-Louis Laplane, qui constitue le plus important récit de la période par un protagoniste ${ }^{14}$. En 2009, dans une étude consacrée aux sans-culottes marseillais, centrée principalement sur les années 17911793, au terme d'une tendance historiographique marquée par une prise de distance avec les sources du début de la Révolution, Michel Vovelle en vient à résumer l'année 1789 à Marseille à une insurrection le 23 mars, consécutive à des placards invitant les ouvriers à s'assembler, sans noter que ces assemblées n'eurent simplement pas lieu, et à l'altercation violente de la Tourette, qui aurait fait 40 morts, quand les sources parlent de 40 blessés ${ }^{15}$. Il décrit l'action politique des protagonistes de cette année en recourant à la catégorie de «foule », puisqu'il reviendrait à la Révolution d'avoir organisé politiquement les citoyens, dans les clubs et les sections, pour «l'affrontement des partis » à Marseille ${ }^{16}$. Pour conclure cette mise au point sur l'historiographie, on notera que les corporations en sont pratiquement absentes.

Pourtant la foule n'est pas la seule modalité d'action des citoyens en 1789. Les communautés de métier constituent la forme d'organisation

(13) Entre autres : Jules VIGUIER, op. cit., p.108, qui annonce la création d'un garde nationale le 23 juillet à Marseille, quand elle ne le fut qu'en février 1790. Adolphe CRÉMIEUX, « Le particularisme municipal à Marseille en 1789 », La Révolution française, 1907, p. 196, commet un contresens complet sur la délibération du 29 décembre 1788, et invente même une "révolution municipale" qui aurait eu lieu en septembre 1788. Paul MASSON (dir.), Encyclopédie des Bouches-du-Rhône, T.14, Paris / Marseille, Honoré Champion / Archives départementales des Bouches-du-Rhône, 1926, p. 252, avance que le conseil des trois ordres se serait réuni jusqu' au 24 mai alors qu'il s'agit du 15 mai et qu'une deuxième révolution municipale aurait eu lieu le 20 juillet quand c'est le 18 juillet. Raoul BUSQUET, Histoire de Marseille, Paris, R. Laffont et J. Laffitte, édition augmentée de 1998, p. 273-274, sous-entend que les loges maçonniques ont eu un rôle à Marseille dans le début du processus révolutionnaire, quand aucune source, et particulièrement celles des archives du fonds maçonnique conservées à la BNF, ne permet d'étayer cette thèse.

(14) Monique CUBELls, « Marseille au printemps de 1789 : une ville en dissidence », Annales du Midi, 1986, p. 67-91. Id., Les horizons de la liberté. Naissance de la révolution en Provence, 17871789, Aix-en-Provence, Edisud, 1987 ; Jean-Louis LAPLANE, Journal d'un marseillais, Marseille, J. Laffitte, 1989, édité par Gérard FABRE et Véronique AUTHEMAN.

(15) Michel Vovelle, Les sans-culottes marseillais, Aix-en-Provence, Presses universitaires de Provence, p.139-142. Les sources sont les suivantes : récit de Mézières, ancien officier au corps-royal de l'Artillerie, imprimé à Marseille en 1790, p. 34, BM Marseille, qui parle de quarante blessés et deux morts dans la foule ; deux morts signalés aussi dans « quès-à-co ? », p. 6, AD Bouches-du-Rhône $16 \mathrm{~F}$ 34 ; un mort dans « siège de la Tourrette », imprimé à Lausanne, p. 10, AD Bouches-du-Rhône, $16 \mathrm{~F}$ 34 .

(16) Ibid., p.143 : la mobilisation marseillaise serait « inorganisée » avant 1789, et « semiorganisée [...] à l'instigation véritable ou supposée du Club » en 1790. 
politique que les maîtres ont utilisée pour lutter. Dans une ville d'Ancien Régime, c'est même la principale forme d'organisation politique légale et elle concerne des effectifs bien plus nombreux que les corps de ville, $20 \%$ des hommes de plus de vingt ans à Marseille ${ }^{17}$. Ce travail vise donc à décrire le processus révolutionnaire à Marseille en 1789 en tenant compte de cette force que représentaient les maîtres assemblés.

Les fonds locaux et nationaux ont été utilisés. Ils se recouvrent relativement peu, ce qui justifie et même implique la nécessité d'y recourir conjointement. Les procès verbaux $(\mathrm{PV})$ de délibération des corporations sont souvent conservés dans la série $\mathrm{E}$ des fonds départementaux, ce qui est en partie le cas à Marseille. Mais ce sont dans les minutiers de notaires qu'a été retrouvée la majeure partie du millier de PV utilisés dans cette étude, dont 370 pour 1789, sources inédites pour la plupart. Ont également été utilisés : les correspondances des échevins, les délibérations municipales, les archives des corporations, qui donnent entre autres des règlements, des pièces de compte, des recueils d'imprimés, les délibérations de la chambre de commerce, les fonds maçonniques, les fonds des comités de l'assemblée nationale, les séries relatives à la convocation des États généraux, les fonds du service historique de la défense ${ }^{18}$. Cette présentation des sources traduit une volonté de documenter l'espace social dans la plus grande extension possible.

La première phase du processus révolutionnaire à Marseille s'étend de décembre 1788, où l'on assiste au début de la politisation de la municipalité et d'une partie de la ville autour de l'enjeu des modalités de convocation aux États généraux, à février 1790, lors de la mise en place de la nouvelle municipalité révolutionnée par l'Assemblée nationale. Le bilan historiographique a montré que nous dépendons principalement, pour cette période, d'une étude de 1839. Ceci explique un caractère parfois narratif et descriptif de ce travail, qui vise à faire le point sur un certain nombre d'événements clés de la période. Et surtout, à introduire dans le tableau des événements, un acteur important, les communautés d'arts et métiers.

(17) Cf. ci-dessous.

(18) AMM ; AA 70, BB 215 à BB 224, BB 287 à 291, BB 358 à 360, 1BB 3313 à 3360, EE 62 à 67, FF 305 à 312, FF 396-399, HH 386 à 440. AD BDR : B 3341 à 3465, C 2378 à 2399, C 2752, C 4110, $240 \mathrm{E}$ et 250E, 72 minutiers de notaires dans la série E, 16 F 34. BM Marseille, recueil Michel de Léon, 29 volumes, série Xd contenant des brochures, almanach Grosson, tableau de Marseille d'Achard, journal de Ferréol Beaugeard. CCM, A 24 et 25, B 20. AN, B III 82, BB 30 14, 15 et 91, C 19, D XXIX 53 à 55, D XXIXbis 1 à 3, H1 1358 et 1359. SHD, A4 LV et LVI, Xb 40, 74, 98, 81, Ya 355*, 517 et 527*. BNF, toutes les cotes concernant les loges marseillaises dans le fonds maçonnique pour l'Ancien Régime, notamment le livre d'architecture de la loge de la triple union, FM3 387. 
Outre la question des corporations, le cas marseillais permet d'enrichir le schéma classique concernant les révolutions municipales en 1789. Les corporations ne sont généralement pas présentées comme des acteurs ayant participé à ce processus de révolutions municipales à travers le royaume. Elles sont pratiquement absentes de l'article que Daniel Ligou a consacré en 1960 à la révolution municipale, que Maurice Genty présentait en 1989 comme encore « très neuf », et absentes aussi du travail de Lynn Hunt $^{19}$. Pourtant, ce qui affleure dans quelques études, c'est bien le fait que les corporations ont été un acteur important du rapport de force et de la dynamique conduisant à la transformation des municipalités d'Ancien Régime, avec des variations spatiales qui restent à établir ${ }^{20}$.

Par ailleurs si, à Marseille, la pré-révolution ne fut pas très animée, dans d'autres villes, les corporations participèrent activement à la politisation de cette période ${ }^{21}$. De la fin de l'année 1788, aux révolutions municipales de 1789, ce sont en fait les mêmes acteurs qui luttent, autour de la question de l'organisation du pouvoir politique. Il conviendrait donc, dans les villes, de ne pas séparer l'étude de la pré-révolution de celle des révolutions municipales. La borne aval de ce processus de reconfiguration du champ du pouvoir dans les villes consiste en la mise en place des nouvelles municipalités suite à la loi du 14 décembre 1789 .

Pour poser les bases d'une compréhension de la participation des corporations au processus révolutionnaire, il est nécessaire de décrire le monde corporatif marseillais à la veille de la Révolution, en précisant

(19) Daniel Ligou, « À propos de la révolution municipale », Revue d'histoire économique et sociale, 38, 1960, p. 146-177; Maurice GENTY qui actualisa la bibliographie de la 7e édition de Georges LEFEBVRE, La Révolution française, Paris, Presses universitaires de France, 1989, p. 713. Lynn A. HunT, « Committees and Communes : local politics and national revolution in 1789 », Comparative studies in society and history, Vol. 18, N³, 1976, p. 321-346.

(20) Outre le travail essentiel de Steven L. KAPLAN, La fin..., op. cit., voir : pour Dijon, Auguste COCHIN et CHARPENTIER, La campagne électorale de 1789 en Bourgogne, Paris, 1904 ; pour Arles, Monique CuBELLS, « La ville d'Arles au printemps de 1789 », dans Jean SENTOU, Révolution et contre-révolution dans la France du Midi, Toulouse, Presses universitaires du Mirail, 1991, p. 29 ; Pierre SERnA, Antonelle, aristocrate révolutionnaire (1747-1817), Paris, Éditions du Félin, 1997, p. 117-125; pour Bordeaux, Michel LHÉRITIER, La révolution à Bordeaux dans l'histoire de la Révolution française. Tome 1, Paris, Presses universitaires de France, 1942, p. 111-139. D'une manière générale, les corporations sont abordées de manière allusive, avec très peu de recours à leurs archives propres, et moins encore aux archives notariales, qui peuvent pourtant se révéler être une source importante de l'histoire politique, comme le montre ce travail.

(21) C'est le cas au moins à Dijon, Toulouse, cf. Steven L. KAPLAN, La fin..., op. cit., p. 371376, où Steven Kaplan pointe le défaut de connaissances précises de la part prise par les corporations dans le processus révolutionnaire. Un travail de repérage des sources effectué dans les fonds municipaux et départementaux des villes de Dijon, Lyon, Saint-Étienne, Aix-en-Provence, Toulon et Toulouse, confirme l'hypothèse que les corporations ont joué un rôle politique dans les villes où les réformes de Turgot en 1776 ne les avaient pas affaiblies, soit Dijon, Aix-en-Provence, Toulon, Toulouse, même si à Toulon les sources sont lacunaires. 
l'influence qu'a pu avoir le courant réformateur au XVIII ${ }^{\mathrm{e}}$ siècle, et notamment les réformes de 1776. L'organisation politique des corporations sera décrite et on pourra ainsi mesurer l'écart avec le cas parisien et préciser la culture des maîtres en matière d'organisation politique. Ces structures posées, les événements de la longue année 1789 seront détaillés. Il sera alors temps de traiter la question du rapport entre les prises de position politiques des corporations et leur caractérisation socio-économique. Autrement dit, le positionnement des maitres dans l'espace social est-il au principe d'une hétérogénéité de positionnements politiques?

\section{Les corporations à la veille de la Révolution}

\section{Un monde corporatif pleinement constitué}

Les réformes successives des corporations initiées par Turgot en février 1776 n'ont eu ni application ni incidence à Marseille ${ }^{22}$. De plus, à la fin de l'Ancien Régime, les corporations ne sont l'objet d'aucune attaque en règle, ni de la municipalité, ni du Parlement, ni de l'intendant. C'est donc un monde corporatif pleinement constitué qui rentre en interaction avec le processus révolutionnaire en 1789. Par ailleurs, le début du processus révolutionnaire est un bon révélateur de la manière dont ceux qui exercent des pouvoirs perçoivent les corporations. Contrairement aux catégories amorphes de la «foule » ou du «peuple séditieux », les corporations (et leurs maitres) sont nommées, connues, classées, et reconnues comme groupes ayant une existence légitime dans l'organisation urbaine. Les échevins, le commandant militaire de la province, ou Mirabeau, ne cessèrent de les solliciter en 1789 pour participer aux deux gardes bourgeoises ou pour asseoir les impositions nouvelles. Quand il fallut les convoquer pour élire les députés aux États généraux, non seulement aucune ne fut oubliée, mais les échevins poussèrent même le zèle jusqu'à convoquer d'infimes groupes qui déclinèrent l'invitation, disant ne pas faire corps. De plus, alors que les règlements du roi pour la convocation des États généraux ne prévoyaient pas que des doléances soient rédigées dans les assemblées primaires, les échevins leur demandèrent de rédiger des cahiers. Mauvais calcul politique et signe révélateur que les corporations étaient prises au sérieux.

(22) Les édits de février et août 1776 ne furent jamais enregistrés au Parlement d'Aix. Pour d'autres provinces, et en premier lieu dans le ressort du Parlement de Paris, voir Steven L. KAPLAN, $L a$ fin..., op. cit., p.128-162. 
À Marseille, tous les maîtres pouvaient participer aux assemblées de leur communauté, ce qui n'était pas le cas à Paris ${ }^{23}$. Combien de maîtres trouvait-on dans ces corporations à Marseille en 1789 ? En l'absence de sources fiscales ou de dénombrements systématiques, le recoupement des listes de noms des participants aux assemblées permet, en éliminant les doublons, de constituer un recensement des effectifs des communautés. En 1789, il y avait à Marseille environ 8000 maittres, pour une population totale de 120000 personnes ${ }^{24}$. En utilisant un sex-ratio de 1, et une proportion de moins de 20 ans de $34 \%$, on trouve que les maîtres représentent $20 \%$ des hommes de plus de vingt ans, ville et terroir compris ${ }^{25}$.

Les communautés sont assez diverses. Certaines sont grandes (un millier de portefaix), d'autres petites (une dizaine d'arquebusiers) ${ }^{26}$. Certaines ne concernent qu'un métier, d'autres plusieurs : les constructeurs font communauté avec les charpentiers, rémolats, poulieurs et machinistes. Par ailleurs, il existait dans l'Ancien Régime une distinction entre les communautés d'arts libéraux et d'arts mécaniques, les premières étant mieux considérées que les secondes ${ }^{27}$. Lors des fêtes et réceptions, pour lesquelles des défilés étaient organisés, les rangs des corporations étaient réglés, et ces préséances pouvaient être l'enjeu de luttes ${ }^{28}$. Du point de vue des conditions nécessaires pour pouvoir faire partie du conseil municipal ou

(23) Steven L. KAPLAN et al., «Idéologie ... », op. cit. Voir par comparaison Bernard GALlinATO, Les corporations à Bordeaux, op.cit., p.143-161. Sur les corporations à Marseille, il n'existe pas d'étude d'ensemble, et pratiquement aucune étude publiée. Augustin FABRE, Les rues de Marseille, Marseille, Camoin, 1867-1869, a traité des communautés des pêcheurs, portefaix, avocats et huissiers. Michael SONENSCHER, The hatters of eightennth-century France, Berkeley, University of California Press, 1987, a étudié celle des chapeliers en France, avec un travail d'archives sur Marseille.

(24) Le recensement des effectifs des maîtres pour l'année 1789 a été fait à partir des minutiers des 28 notaires de Marseille qui ont été dépouillés systématiquement, qui donnent 370 PV d'assemblée pour 58 corporations. Sur le dénombrement de la population marseillaise, voir Charles CARRIÈRE, Négociants marseillais au XVIII siècle, Marseille, Institut historique de Provence, 1973, p. 193-211. cit., p. 40

(25) Cf. le dénombrement de 1765, AMM, FF 187 p. 370 et suivantes ; Michel VovelLE, op.

(26) Les portefaix étaient en conflit avec les robeirols, qui disputaient leur travail, mais qui ne formaient pas comme les portefaix une communauté, et ne prirent aucune part identifiable aux luttes politiques. Les arquebusiers et les armuriers ont des statuts datés du 15 avril 1716, enregistrés au Parlement le 24 décembre 1725 (AMM, FF 307 f 85, AD BDR, BB 3394 f589, AMM, HH 389). En 1789, ils ont un syndic, Esprit Sic, qui est d'ailleurs élu député du corps (AD BDR, 364 E 505). Ce genre de petite corporation n'est pas une spécificité marseillaise.

(27) Voir Nicolas GUYOT, Répertoire universel et raisonné de jurisprudence, Paris, 1775 , article « arts et métiers ». Lors de la désignation des représentants aux États généraux, la qualité d'art libéral conféra par exemple l'avantage de pouvoir élire deux députés pour cent votants dans les assemblées primaires, quand les arts mécaniques ne purent en élire qu'un seul. Mais ces catégories étaient relativement floues. Elles furent notamment l'enjeu d'une lutte au moment de l'élection des députés.

(28) AMM, FF 187 p.174-176, et les registres du cérémonial dans la série AMM AA. 
de l'échevinat, la hiérarchie des groupes est assez claire ${ }^{29}$. Elle part des négociants non nobles, ou des marchands qui font le commerce en gros, puis ce sont les bourgeois ou les marchands qui ont cessé de tenir boutique ouverte depuis trois ans et qui ne font pas de commerce, puis les marchands tenant boutique ouverte, puis les médecins. L'assesseur est nécessairement un avocat. Aucune autre corporation que celle des marchands, des médecins ou des avocats ne peut conférer à un individu le privilège de participer à la municipalitée ${ }^{30}$. Les corps de métier n'avaient plus accès au conseil de ville depuis le XII ${ }^{\mathrm{e}}$ siècle $^{31}$.

\section{La culture des maîtres en matière d'organisation politique}

Les corporations, grâce à leur entrée dans le processus révolutionnaire, constituent un lieu exceptionnel, car précoce, pour sonder les conceptions d'une partie des maîtres en matière d'organisation du pouvoir politique. Autrement dit, un terrain permettant de préciser la culture politique d'une fraction importante du peuple urbain, en observant les maîtres dans leurs assemblées. C'est un terrain relativement sûr, notamment par rapport aux tentatives de reconstitution de la culture politique par l'intermédiaire de l'analyse des doctrines mises en circulation, où se pose toujours la question de savoir qui les a lues et comment ${ }^{32}$.

L'assemblée est clairement un outil de prise de décision qui fait consensus parmi les maîtres. Le bien fondé de se rassembler pour prendre des décisions n'est jamais discuté. Au contraire, les conflits de pouvoir avec les conseils dont sont dotées certaines corporations indiquent que l'organe souverain est à la fois conçu comme devant être et comme étant de fait l'assemblée ${ }^{33}$. Par ailleurs, selon les maîtres, quelles sont les conditions de légitimité d'une assemblée d'élus auxquels est délégué un pouvoir? Les horlogers avancent qu'elle doit être suffisamment nombreuse, et de composition homologue à celle des électeurs ${ }^{34}$. Les cahiers de doléances

27.

(29) Règlement municipal de 1717 dans AMM, BB 384, celui de 1766 dans AD BDR, 1 F

(30) Noter que les négociants ne forment pas une communauté.

(31) George LESAGE, Marseille angevine; recherches sur son évolution administrative économique et urbaine de la victoire de Charles d'Anjou à l'arrivée de Jeanne ler (1264-1348), Paris, E. De Boccard, 1950.

(32) Roger CHARTIER, Les origines culturelles de la Révolution française, Paris, Seuil, 2000, p. 196.

(33) Patrons pêcheurs, le 2 août 1789, BMM, recueil Michel de Léon, T.XXIII, p. 224 : « il faut des conseils, c'est à dire, des assemblées représentatives aux grandes agrégations politiques, \& là où il est impossible que tous les membres concourent directement à la chose publique; mais on ne persuadera jamais qu'une communauté de 200 personnes ait besoin de se choisir des mandataires ».

(34) AD BDR, 370 E 63 f³57v. 
fourmillent d'arguments similaires. La légitimité de l'élection n'est jamais discutée comme mode d'accès aux charges. L'élection « libre » est perçue comme productive d'un « esprit de zèle et de patriotisme $»^{35}$.

Les maîtres prennent leurs décisions soit à l'unanimité, soit à la majorité des voix. Le principe majoritaire est-il perçu comme une manière robuste de trancher les conflits (politiques) pacifiquement ? Dans la majorité des cas il semble que oui, et on trouve parfois mention de scrutins assez serrés, où la majorité s'impose sans l'intervention d'autorité extérieure pour légitimer la décision par un autre moyen.

La rotation des charges et l'impossibilité d'exercer de multiples fois la même charge sont discutées. Par exemple, les calfats avaient voté en 1783 l'interdiction d'être premier prud'homme plus d'une fois dans sa vie. Or un maitre calfat s'était pourvu en opposition contre cette délibération. Le cas fut examiné en assemblée le 13 juillet 1789. Les conclusions de deux avocats au Parlement furent exposées ${ }^{36}$. Ils développèrent l'idée que la rotation obligatoire des charges était en contradiction avec le principe électif :

« quelques purs que soient les motifs de cette délibération qui semble n'avoir eu pour objet que d'apeller un plus grand nombre de membres à l'administration du corps, [...] on ne peut se dissimuler qu' [elle] ne gene la confiance, et ne soit contraire à la liberté qu'ont tous les membres de donner leur voeu à ceux qui ont déjà fait preuve de zele, de talent et d'experience dan quelqu'une des places de prud'homme».

La consultation recommanda au corps d'accepter la cassation de la délibération de 1783, et l'assemblée accepta ${ }^{37}$.

Toutes ces questions d'organisation politique, la pratique de l'assemblée, l'usage de l'élection, la rotation des charges et surtout la délégation des pouvoirs, ont été des enjeux des luttes révolutionnaires. Il n'est pas inutile de connaître, sur ces points, l'état culturel dans lequel les maîtres abordèrent l'année 1789. Les pratiques d'Ancien Régime montrent que les maîtres étaient attentifs à la question du pouvoir et des procédures qui permettaient de le réguler.

(35) « Libre » par opposition au vote portant sur une personne choisie à l'avance.

(36) AD BDR, 363 E 397.

(37) Il est intéressant de noter que, selon Bernard MANIN, Principes du gouvernement représentatif, Paris, Flammarion, 1996, p. 49, « Les Athéniens avaient conscience du conflit potentiel entre le principe électif et le principe de rotation, c'est pourquoi rien n'interdisait d'exercer plusieurs fois de suite la même magistrature élective »; c'est la position défendue ici par les avocats. 


\section{La Révolution à Marseille en 1789}

\section{Une politisation limitée (1788 - février 1789)}

À Marseille, le débat sur les modalités de convocation pour les États généraux ne fut pas particulièrement vif ${ }^{38}$. Les premiers à prendre position furent les négociants, par l'intermédiaire du bureau de la chambre de commerce. Le but énoncé dans la délibération du 5 novembre 1788 était « d'avoir des députés aux etats generaux pour representer le commerce ». Pour cela, ils tenaient une correspondance avec le ministre de la Marine, le comte de la Luzerne, et de La Tour, l'intendant de la province ${ }^{39}$. Le conseil de ville prit position le 29 décembre $1788^{40}$. Il préconisa que l'élection pour députer aux États généraux soit faite dans un conseil municipal « renforcé », composé de membres des trois ordres avec, pour le tiers état, des députés élus parmi tous les propriétaires et tous les citoyens appartenant à un corps ou à une communauté.

Le règlement de convocation que fit paraître le roi le 24 janvier 1789 tranchait les multiples débats sur l'organisation des États généraux, et ne suivait pas les demandes du conseil de ville. L'article 26 du règlement royal prescrivait, pour le tiers état, la convocation des « corporations [...] et tous les autres citoyens réunis par l'exercice des mêmes fonctions et formant des assemblées ou des corps autorisés ${ }^{41}$. C'est ce que firent les échevins le 13 mars, en appelant 78 communautés à participer aux élections des députés aux États généraux ${ }^{42}$.

(38) Le conseil du roi avait rendu un arrêt le 5 juillet 1788. Cet arrêt a fait l'objet d'interprétations exagérées, par exemple : Jean EGRET, La Pré-révolution française (1787-1788), Paris, Presses universitaires de France, 1962, p. 325, selon lequel cet arrêt « avait accordé, en fait à la presse politique, une large liberté, puisqu'il conviait tous les Français à donner leur avis sur les moyens de faire des États généraux promis, « une Assemblée vraiment nationale par sa composition comme par ses effets » ». Or l'arrêt ne demande jamais à « tous les Français » de prendre position. Au contraire, seules les administrations provinciales sont explicitement désignées comme devant rendre des avis sur la question, les administrations municipales étant cantonnées dans un rôle de collecte d'informations sur les formes des États généraux de 1614, et de transmission de ces informations aux administrations provinciales. Les échevins marseillais ont précisément suivi cette voie, à la nuance près qu'en tant que Terre Adjacente en Provence, ils transmirent leurs recherches non pas aux États de Provence, mais directement à l'administration royale. Par ailleurs, le régime de liberté de la presse n'était en rien modifié à Marseille. Les écrits politiques circulent sous le manteau, cf. Jean-Louis LAPLANE, op. cit., p.73, et sont parfois saisis, comme les deux imprimés « avis aux provinces » et « profession de foi d'un militaire », et condamnés par le lieutenant général civil de la sénéchaussée de Marseille, BNF, Lb39 6504.

(39) CCM, B 20 p.83.

(40) AMM, BB 223, AN, B 30 91. C'est sur cette assemblée qu'Adolphe CRÉMIEUX fait un contresens, art. cit. Bien noter que l'arrêt du conseil du roi du 5 juillet 1788 ne demandait pas aux municipalités de prendre position sur la convocation aux États généraux.

(41) Voir le T.1 des Archives Parlementaires.

(42) AMM, BB 290, f ${ }^{\circ} 20 \mathrm{r}$. 
Selon Steven Kaplan, « [en province], les édiles manipulèrent les convocations afin de réduire la présence ou l'influence corporative dans la rédaction des cahiers $[\ldots] \gg^{43}$. Cela ne semble pas être le cas à Marseille, puisque les échevins ont convoqué tous les corps de métier dont nous avons conservé quelques archives. De plus, les échevins ont relayé sans protester, la mise à l'ordre du jour des assemblées des communautés, décidée par le lieutenant général civil de la sénéchaussée, de la rédaction d'un cahier « de ses plaintes \& doléances », ce qui n'était prévu dans aucun règlement du roi, qui réservait cette rédaction à l'assemblée générale du tiers état des bailliages et sénéchaussées ${ }^{44}$. S'ils avaient voulu museler les communautés, ils n'auraient certainement pas relayé cette demande. Ils auront d'ailleurs quelques raisons de le regretter.

\section{Les corporations et la foule gagnent une bataille : première révolution municipale et autres conquêtes}

Les élections pour les États généraux commencèrent le 15 mars, par l'assemblée des auffiers, et finirent le 29 mars par celle des meuniers. L'essentiel des assemblées se tint néanmoins entre le 15 et le 23 mars. En tout, on compte 72 assemblées pour 5172 maîtres, au cours desquelles furent élus les députés pour l'assemblée du tiers état de la ville ${ }^{45}$. Les maîtres y discutèrent aussi des doléances, qui furent votées ${ }^{46}$. Elles étaient unanimes sur au moins deux points. Premièrement, elles réclamaient la réforme du régime des impositions, qui portaient uniquement sur les comestibles, à l'avantage de tous les possédants de biens ${ }^{47}$. Deuxième revendication unanime, l'élargissement du conseil municipal : les corporations voulaient y députer directement pour prendre part au pouvoir municipal.

Par ailleurs, de nombreux cahiers de doléances avaient été imprimés et mis en circulation. Les délibérations autour de ces doléances, qui ont rassemblé à Marseille au moins 7500 personnes, et leur impression, sont des événements essentiels. Voici comment les échevins commenteront, plus tard, le 19 juin, la mise en circulation des doléances :

(43) Steven L. KAPLAN, La fin... op. cit., p.364.

(44) AN, B III 82 p.58.

(45) Ce chiffre ne prend pas en compte les non-corporés, négociants, bourgeois, ménagers et les 220 ouvriers assemblés le 29 mars, soit en tout 2283 personnes supplémentaires non corporées.

(46) Joseph FOURNIER, Cahiers de doléances de la sénéchaussée de Marseille pour les États généraux de 1789, Marseille, 1908.

(47) Revendication qui fait suite à plus d'un siècle de luttes populaires contre les fermiers, cf. Jean NICOLAS (dir.), La rébellion française, Paris, Gallimard, 2008. La majorité des corporations demandent également la suppression pure et simple des fermes de la ville. 
«Lors de la convocation des etats généraux, le peuple et toutes les corporations formèrent le premier article de leurs plaintes sur cette imposition municipale. La liberté de la presse fit distribuer des déliberations de plusieurs corporations qui contenoient les déclamations les plus violentes sur l'imposition du pain et de la viande. Tel a été le prétexte ou la cause des mouvemens populaires qui ont agité Marseille le 23 mars dernier ${ }^{48}$.

Car que sont des doléances? Un moyen de fixer les idées par écrit, sorte de programmes politiques, débattus et votés, en assemblée, accomplissant le passage des volontés individuelles à la volonté collective tranchée à la majorité. Ces doléances ont servi de ligne de conduite tout au long de l'année 1789.

Le 23 mars, l'essentiel des assemblées de corporation étaient terminées. Après un voyage à Marseille qui fut un triomphe, Mirabeau était retourné à Aix. Une révolte fut déclenchée, qui se poursuivit le lendemain ${ }^{49}$. Ces journées des 23-24 mars 1789 sont généralement qualifiées d'émeutes frumentaires $^{50}$. Cependant, des sources indirectes permettent de reconstituer les revendications des émeutiers. La première décision prise par les échevins le 23 mars fut de diminuer les prix du pain et de la viande, ce qui était sans ambiguïté une revendication des émeutiers. La deuxième décision prise par les échevins fut d'appeler les députés des corporations à participer au conseil de ville. Or c'était une revendication unanime des corporations. En conséquence, un premier conseil renforcé des députés des corporations se tint le 24 mars. La troisième décision fut d'abolir les fermes de la ville. C'était également une revendication des corporations et certainement des émeutiers, puisque ces derniers attaquèrent le 23 mars la maison du régisseur des fermes. Le 26 mars, les 210 personnes participant au conseil de ville, où se trouvaient 114 députés des corporations, décidèrent de nommer 24 commissaires pour mettre en place un nouveau régime d'imposition en remplacement des fermes abolies.

(48) AMM, BB $290 \mathrm{f}^{\circ} 112 \mathrm{v}$.

(49) Voyage de Mirabeau du 17 au 19 mars, Jean Louis LAPLANE, op. cit., p. 90 ; l'impression des cahiers de doléances est signalée dans AMM, BB 290 f $112 \mathrm{v}$; ces imprimés se trouvent réunis dans le recueil Michel de Léon (BMM), et ont été utilisés par Joseph FoURNIER, op. cit.

(50) Par exemple, Monique Cubells, Les horizons de la liberté, op. cit., considère p. 94 que les émeutes ont pour enjeu principal les «subsistances », et pour enjeu secondaire la « politique ». Les éditeurs du journal de Laplane ne commentent que la hausse des prix des denrées : note 2 p. 117 ; de même Gérard-José MATTEI, Marseille, une cité face à la Révolution, 1788-1792, Thèse de doctorat d'État en droit, 1978, p. 162. François-Xavier EMMANUELLI, « Aux origines de la Révolution en Provence », $A H R F, 254,1983$, p. 562, a nuancé l'intensité de la hausse des prix. 
Il est clair que les décisions ont été prises sur revendication des insurgés et des corporations. Elles ont un caractère politique très marqué, puisqu'elles touchent d'une part à la distribution du pouvoir politique dans la ville, et d'autre part à la redistribution des richesses par l'intermédiaire des impôts. L'insurrection des 23-24 mars n'est pas seulement une émeute frumentaire. Ce qui la distingue et la caractérise, ce sont ses implications politiques. La suite des événements va permettre de préciser la signification de ces journées et de hiérarchiser les objectifs poursuivis par les corporations.

La conquête provisoire, par les corporations, de la possibilité d'envoyer des députés au conseil de ville est de première importance. Ce projet était explicité dans la majorité des cahiers de doléances. À l'inverse, dans des villes comme Troyes et Reims, si les corporations y ont aussi écrit des doléances, elles n'y ont pas fait figurer de revendications sur la forme du pouvoir local, et elles n'ont, semble-t-il, tenté aucun coup de force pour participer au conseil de ville ${ }^{51}$. Le fait de formaliser par écrit les revendications n'a pu qu'être une cause facilitante du processus de conquête politique.

À partir de mars, trois objets sont plus particulièrement l'enjeu de luttes. Tout d'abord, la composition du conseil de ville et la répartition $d u$ prélèvement de l'impôt. Les corporations relient ces deux enjeux, et considèrent comme nécessaire de changer la composition sociale du conseil pour avoir une garantie que l'impôt soit plus équitablement réparti. Le troisième enjeu de lutte, où les corporations sont encore impliquées, concerne la composition ainsi que l'organisation de la garde bourgeoise.

\section{Une nouvelle garde de ville (23 mars - 20 mai 1789)}

En effet, la constitution autonome d'une garde bourgeoise était une nouveauté issue des 23 et 24 mars. Son rôle lors de ces deux journées a été de protéger les sorties des échevins et certaines propriétés attaquées ou menacées. Marseille avait bien une garde de ville, qui comptait une centaine de personnes, et qui était notoirement insuffisante ${ }^{52}$. Concernant cette nouvelle garde, ses officiers étaient principalement des notables, et

(51) Lynn HunT, Revolution and urban politics in provincial France. Troyes and Reims, 1786-1790, Stanford, Stanford Univ. Press., 1978.

(52) Un règlement de 1787 prévoit 128 personnes divisées en 4 compagnies, soldées par la commune. Un impôt spécial était levé pour cela sur les «magasins, boutiques, remises \& ecuries qui sont dans l'enceinte de ladite ville », AMM, EE 63 et AMM, EE 64. Sur l'insuffisance de la garde, voir AMM, BB 358 f39r. 
son recrutement se caractérisait par la présence des portefaix et de jeunes gens issus de la bourgeoisie ${ }^{53}$.

L'implication des corporations dans la nouvelle garde peut aussi être documentée. En effet, les échevins craignaient cette force armée qu'ils ne semblent pas contrôler. Le 9 avril au soir, ils réunissent à l'hôtel de ville " les jeunes citoiens qui font la garde de la ville et les membres des diverses corporations ». Les échevins exposent alors qu'ils ont

«mis pour chef de divisions, ceux des citoiens, qui pourroient etre les plus agreables à la bourgeoisie, et à qui celle cy avoit deja voulu donner le titre de capitaine ». Et « les jeunes citoiens ont accueillis avec enthousiasme notre projet, ils l'ont tout de suite mis à execution, mais les membres des corporations qui étoient à l'hôtel de ville au moins au nombre de 400 demandoient beaucoup d'explications, faisoient des reflexions qui pouvoient être de dangereuses conséquences $»^{54}$

Une opposition des maîtres se dessine. Le lendemain, 10 avril, les échevins font « venir à l'hôtel de ville les prieurs des corporations les uns après les autres, pour savoir d'eux le nombre d'hommes qu'ils peuvent fournir pour la formation de la garde bourgeoise $\gg^{55}$.

Quelle est alors la réaction des corporations? Du 11 au 19 avril, 29 corporations tiennent assemblée, pour un total de 1321 maîtres présents. Si toutes les délibérations soutiennent la nécessité d'une garde bourgeoise, seuls les « rôtisseurs \& pâtissiers » et les ferblantiers acceptent sans condition d'y participer en l'état. Les autres corporations émettent des réserves aux sollicitations des échevins. La plus courante (23 occurrences), qui rompt avec les pratiques établies, consiste à confier aux volontaires de la garde la possibilité d'élire les officiers. Autre rupture, le refus de servir en tant que corps « vu les contestations qui s'eleveroient a raison des preseances et des inconvenients qui pourroient resulter des disputes qui pourroient s'élever à des heures indues » $(18 \text { occurrences })^{56}$. Ensuite, 17 corporations acceptent de servir dans la garde à condition qu'il n'y ait pas de « distinction d'état [pour ne former] qu'une seule classe de patriotes

(53) Monique CUBELls, « Marseille au printemps de $1789 »$ op. cit. Les sources suivantes confirment la présence des portefaix et des maçons : AMM, EE 65 et 66 ; BMM recueil Michel de Léon, T14 ; AMM, BB 290 f 97r.

(54) AMM, BB 290 f56v.

(55) Ibid.

(56) Tonneliers, 13 avril, AD BDR, 367 E 287, je souligne. En temps de crise, il semble que l'usage était de lever une garde exceptionnelle composée de volontaires pris dans les corporations, avec les problèmes de préséances typiques des luttes de classements entre communautés, voir AMM, EE 72. 
destines a antretenir la paix, a perpetuer le bon ordre, et a assurer la tranquilité des habitants $»^{57}$. Quant aux hôtes et cabaretiers, ils en appellent à la décision du conseil des trois ordres, déniant par cela toute légitimité au règlement et aux décisions prises par les échevins seuls.

Les échevins, qui ont besoin d'une garde pour faire régner l'ordre, s'accommodent de cette innovation issue des journées de mars, mais échouent donc dans leur volonté de la reprendre en main. Par ailleurs, un certain nombre de Marseillais font jouer leurs relations à la Cour et demandent l'intervention du gouvernement pour casser cette garde. Puis, le 15 mai, Caraman, le commandant militaire de la province dit aux échevins avoir reçu « un courrier de la cour, qui [1]' ordonne de terminer par les moiens les plus courts et les plus positifs les troubles qui agitent votre ville $»^{58}$. Caraman, de concert avec les échevins, met finalement ses ordres à exécution, et entre dans la ville le 20 mai ; ses troupes restent stationnées hors les murs, en vertu des privilèges de la ville et pour ne pas tendre inutilement la situation $^{59}$. Il casse alors la garde, nomme un nouvel étatmajor opposé aux changements, et restaure la forme légale du conseil de ville, suspendue depuis le 24 mars.

\section{La lutte autour de la composition du conseil de ville et la contre-révolution de mai 1789}

En effet, la forme légale du conseil municipal avait été modifiée quand les députés des corporations y avaient été appelés pour la première fois le 24 mars. À ne lire que le registre des délibérations du conseil, tout se passe comme si l'intégration des députés des corporations était une initiative des échevins. Or, ces députés ne ressemblent pas vraiment aux «notables » que La Tour leur suggérait d'incorporer au conseil ${ }^{60}$. Des indices laissent penser que la décision aurait été prise le 23 mars au soir à l'hôtel de ville en présence des députés des corporations et des émeutiers ${ }^{61}$.

Les 24 et 26 mars, deux conseils renforcés sont tenus. Les échevins convoquent celui du 26 en y appelant des membres du clergé, de la noblesse, et d'une manière générale, on assiste à une notabilisation de sa composition, dans l'esprit des conseils de La Tour. Il est cependant trompeur de parler

(57) Menuisiers, 14 avril, AMM, HH 416.

(58) AMM, BB $358 \mathrm{f}^{\circ} 195 \mathrm{v}$.

(59) Elles n'y entreront pas avant le 19 août.

(60) Lettre de La Tour aux échevins le 22 mars, où il leur propose « d'assembler dans un moment critique un conseil » renforcé de notables pour « assurer la tranquilité publique », AMM, BB $358 \mathrm{f}^{\circ} 130$.

(61) Jean Louis LAPLANE, op. cit., p.112; AMM, BB 290, f ${ }^{\circ} 42 \mathrm{v}$. 
d'une fondation d'un conseil des trois ordres les 24 et 26 mars, puisque cela masque le fait que la lutte autour de la composition du conseil de ville ne fait que commencer et que les assemblées suivantes du 26 avril et du 14 mai ont été convoquées par les échevins sous la pression populaire, et non dans le cadre d'un fonctionnement normal. D'un point de vue des pratiques d'Ancien Régime, ces assemblées sont illégales, puisque leur composition n'a pas été sanctionnée par le roi, ou par le Parlement ${ }^{62}$. Par ailleurs, leur convocation est toujours du pouvoir des échevins.

Les échevins prennent l'initiative d'assembler un nouveau conseil renforcé le 13 avril, mais le contrôle de cette séance semble leur avoir échappé. En effet, dans le procès verbal, leur parole reste confinée au début, ce qui est tout à fait atypique. Alors qu'en temps normal ils ont un quasi monopole (de fait) de la proposition, ce n'est plus le cas ici. Notamment, Saurel, député des capitaines de navire, y prend la parole et plaide pour le maintien d'un conseil renforcé, composé de députés des trois ordres, pris selon les proportions actuelles, qui « représenterait [...] parfaitement tous les citoyens ». Et le conseil délibère de demander au roi l'autorisation de transformer ainsi le conseil. Mais les échevins ne firent jamais suivre cette requête, leur correspondance le montre ${ }^{63}$. On voit donc comment les échevins s'opposent à la volonté du conseil, en usant de leur pouvoir et de leurs prérogatives, notamment par leur contrôle de la correspondance officielle. Les deux conseils suivants sont cette fois convoqués non pas sur initiative des échevins mais sur pression populaire, ce qui confirme la défiance des échevins vis-à-vis de ce conseil renforcé.

Après le 20 mai, suite à l'entrée de Caraman, les corporations sont évincées du conseil et ce dernier est restauré dans sa forme d'Ancien Régime, jusqu'à la mi-juillet. Alors, 21 corporations prirent position contre leur éviction du conseil à qui elles déniaient toute légitimité ainsi composé64 . Voilà un nouvel acte de rébellion politique de première importance. Le syndic des magasiniers saleurs exposa ainsi son opinion dans l'assemblée de son corps à propos du conseil de ville. Ce dernier

(62) Sur la jurisprudence concernant les conseils de ville renforcés à Marseille, voir AN, BB 30 91, ainsi que AMM, BB $218 \mathrm{f}^{\circ} 10 \mathrm{v}, \mathrm{f}^{\circ} 14 \mathrm{v}, \mathrm{f}^{\circ} 27 \mathrm{r}$, $\mathrm{f}^{\circ} 70 \mathrm{r}$, f ${ }^{\circ} 81 \mathrm{r}$, délibérations en 1783 du conseil de ville renforcé de notables des quartiers de la ville pour délibérer sur un impôt sur le vin forain. Le conseil renforcé de 1783 ne compte aucun artisan, au contraire de celui issu des journées de mars 1789.

(63) Alors qu'ils demandèrent l'autorisation de l'emprunt de 600000 livres voté le 26 mars, et des 24 commissaires chargés de mettre en place un nouveau régime d'imposition, cf. AMM, BB 290, BB 358 et 359, BB 473.

(64) Les horlogers ont fait imprimer leur délibération du 16 juin, AD BDR, 16 F 34. 
« pour être legal et constitutionel doit être composé non seulement des conseillers municipaux mais encore des representans de chaque corporation $\&$ d'un certain nombre de contribuables choisis parmi les plus notables de la ville, [et] que l'interet du corps exige d'avoir un representant dans le conseil municipal pour concourir à l'établissement de la nouvelle imposition qui sera établie, consentir à la lévée \& statuer avec tous les autres membres du conseil sur la meilleure forme possible pour alléger le fardeau de l'indigent $\&$ eviter le plus de fraix de perception que faire se pourra $»^{65}$.

Sa position est validée par l'assemblée. Le 30 mai, les chapeliers prennent une position homologue :

« dans ces circonstances, nous qui sommes tous citoyens et en partie possedant biens, devont consentir à payer les impositions qui seront etablies par le conseil. Mais il est de l'intéret particulier de notre corporation qu'elle soit réprésentée, ainsi que toutes les autres le reclament, dans le conseil ou se decidera ce nouveau régime d'impots. Ne sommes nous pas une partie de cette cité, \& les corporations ne forment elles pas au moins la moitié de la ville? $»^{66}$.

Le comité des 12 chargé par Caraman de mettre en place une nouvelle imposition, en remplacement du comité des 24 limogé, sollicite les corporations pour obtenir un état de répartition, mais elles refusent invariablement, arguant de l'illégitimité du conseil. Au contraire, les corporations réclament le retour du conseil renforcé et du comité des 24. L'objectif politique central défendu par ces corporations concerne donc la composition du conseil de ville, et la capacité corrélative à y débattre et voter les impositions.

Le 7 juillet, une pétition est déposée chez le notaire Castelanet, intitulée «très respectueuses représentations adressé à [Caraman]. Par différentes corporations d'arts et métiers, grand nombre de chefs de famille $\&$ de citoyens de tous les états de la ville de Marseille ${ }^{67}$. Cette pétition est un plaidoyer pour la participation des députés des corporations à un conseil de ville élargi. On y trouve 793 signataires, dont 118 négociants, 85 maîtres de corporations (dont une trentaine de syndics), 32 clercs, 29

(65) Le 29 mai, AD BDR, 363 E 396.

(66) AD BDR, 367 E 287. Noter que si les ouvriers et compagnons sont ici comptés par le prieur comme faisant partie des corporations, et ce pour les besoins de la démonstration, ils ne participent pas pour autant aux assemblées.

(67) L'original est dans AD BDR, 394 E 84, avec les signatures. 
bourgeois, 23 capitaines de navires, 5 chevaliers de Saint Louis, et hélas, 466 signataires non identifiés. Les négociants y sont surreprésentés, mais vu l'intitulé et le contenu de la pétition, on peut penser qu'elle a été initiée par des corporations.

Cette pétition va connaître un écho important. Elle est le signe d'une convergence de vues entre plusieurs catégories sociales, sur l'enjeu de la composition du conseil. Le 14 juillet, elle est lue et condamnée par le conseil municipal. Mais dans la même séance, Thulis, premier échevin, abdique et réclame «conformément au voeu des citoyens, un nouveau conseil municipal plus nombreux qui serait composé d'un tel nombre que S.M. déterminera et qui sera pris dans un plus grand nombre de classes de citoyens que par le passé ». Et « le conseil [...] a autorisé MM. les maire, échevins, \& assesseurs de faire les démarches nécessaires auprès de S.M. pour obtenir ce nouveau conseil municipal ${ }^{68}$. Ainsi les corporations remportaient une victoire correspondant à leur principale revendication.

\section{Le retour des corporations au conseil : juillet 1789 ou l'autonomie des luttes marseillaises}

Jusqu'à présent, les historiens ont écrit que la restauration du conseil renforcé s'était faite à Marseille en raison des événements parisiens. La nouvelle de l'incendie de la barrière de l'octroi à Paris arrive le 16 juillet au soir selon Laplane, mais le 17, « point de courrier », et l'incertitude grandit quant aux événements parisiens ${ }^{69}$. Alors Caraman décide de convoquer le conseil renforcé pour le 18 juillet $^{70}$. La conjoncture conduit à oublier que les échevins avaient décidé quatre jours plus tôt de restaurer le conseil renforcé, en demandant son autorisation au roi ; et que cette décision a été prise suite au rapport de force construit par les corporations ralliées par certains négociants ${ }^{71}$. Il y a ici une synchronie des calendriers, et un renforcement des effets des actions des protagonistes engagés dans une lutte, chacun dans sa ville. Il semble donc impropre de décrire une révolution municipale à Marseille en juillet comme étant une conséquence des événements parisiens. Premièrement, parce que l'élargissement du

(68) AMM, BB $224 \mathrm{f}^{\circ} 162 \mathrm{r}$.

(69) Jean Louis LAPLANE, op. cit., p.163.

(70) AMM, BB $359 \mathrm{f}^{\circ} 1$.

(71) Noter que les doléances des négociants en mars proposaient une extension du conseil à 90 membres, avec une entrée des députés des corporations et des ménagers, qui devaient composer le tiers du conseil. Autrement dit, les négociants n'avaient pas attendu la première victoire des corporations du 23 mars 1789 pour réclamer, eux aussi, leur entrée au conseil de ville, tout en maintenant leur nombre à 30 députés sur 90 . 
conseil a eu lieu pour la première fois le 24 mars 1789. Deuxièmement, parce que le rapport de force qui aboutit au rétablissement de ce conseil s'est construit avant tout à Marseille même, de mars à juillet. L'arrivée des nouvelles des événements parisiens n'a fait qu'accélérer un événement structurellement acquis.

\section{Suite de la lutte autour de la composition de la garde bourgeoise.}

Concernant la garde bourgeoise, dirigée par des conservateurs depuis fin mai, un affrontement l'opposa le 19 août, sur la place de la Tourette, à des individus que les sources ne permettent pas d'identifier précisément ${ }^{72}$. Le soir même, en violation d'un privilège de Marseille, Caraman fait entrer des troupes royales dans la ville. Le 20 août, une partie du commandement de la garde démissionne ${ }^{73}$. Les corporations sont alors mobilisées par Caraman afin de pourvoir au remplacement des démissionnaires, et pour restaurer le crédit de la garde. La première quinzaine de septembre, un millier de maîtres se rassemblent dans trente et une assemblées pour députer au conseil de ville, afin de désigner les corporations qui devaient élire des capitaines de la garde bourgeoise. Mais huit corporations prennent position contre l'actuel règlement de la garde bourgeoise, en revendiquant que la garde soit organisée par quartiers, avec des officiers élus ${ }^{74}$. Elles refusent d'envoyer des députés au conseil, ou les mandatent pour protester. Puis, fin septembre, à nouveau un millier de maîtres élisent, dans vingt-deux assemblées, capitaines et lieutenants de la garde. La garde n'en tira pas de crédit supplémentaire jusqu'à son remplacement début $1790^{75}$.

\section{La signification de la deuxième révolution municipale d'octobre 1789}

Après le 18 juillet, les corporations ne prennent plus position sur la question de la composition du conseil de ville, puisque l'objectif d'y députer directement semble atteint. En effet, le conseil renforcé par les députés des corporations a été rétabli le 18 juillet suite à la mobilisation des maîtres et

(72) Jean-Louis LAPLANE, op. cit., p.180, les qualifie de « canaille révolutionnaire ». Voir aussi AN, D XXIX 53 et 54 qui donne le procès verbal de l'inspection des prisonniers, avec mention de leurs qualités : c'est sur cette source que reposent les statistiques données par Michel VovELLE, op. cit., p. 140-141.

(73) Démission de 28/60 capitaines de la garde, C. LOURDE, op. cit., p.102, qui interprète l'événement comme une machination des « aristocrates » opposés à la révolution pour permettre l'entrée des troupes de Caraman en ville.

(74) Règlement du 25 mai, modifié le 27 juin, AMM, BB 224 f 154, EE 65.

(75) Faute de sources, on ne peut étudier la composition des volontaires de cette garde, et ainsi vérifier l'implication réelle des maîtres au delà de l'élection des capitaines. 
il se réunit maintenant régulièrement. Caraman a personnellement soutenu sa renaissance. La situation ne semble donc plus être précaire comme elle l'était entre mars et mai, quand les conseils des 26 avril et 14 mai avaient été convoqués sous pression populaire, en toute illégalité vis à vis du pouvoir royal.

Mais, le 3 octobre, d'André, député à l'Assemblée nationale et commissaire du roi arrivé à Marseille le 16 septembre, rend avec Caraman une ordonnance convoquant des assemblées de quartier pour élire des représentants au conseil de ville. Cette opération met fin à la participation des députés des corporations, qui sont remplacés par les députés des quartiers ${ }^{76}$. Ce nouveau conseil composé des échevins, des conseillers de ville ordinaires et des députés de quartiers se réunit régulièrement jusqu'à la mise en place des municipalités selon la loi du 14 décembre 1789, début février 1790.

On ne sait pratiquement rien de ces assemblées de quartiers, hormis la liste des 87 élus, dont 32 négociants, 19 maîtres de corporation, 9 bourgeois, 4 paysans propriétaires, 4 membres du clergé, 3 de la noblesse, 2 courtiers et 2 avocats ${ }^{77}$. Aucune trace de protestation de la part des corporations n'a pu être trouvée, alors que le résultat de l'élection allait contre leur revendication de pouvoir députer directement au conseil. C'est une passe d'armes essentielle, qui n'a pour l'instant jamais été commentée.

En effet, ici se situe la deuxième révolution municipale. La première, initiée le 24 mars, consolidée le 18 juillet, s'était faite en partie sur des principes d'Ancien Régime. Le conseil de ville était un « conseil des trois ordres », et surtout, pour en faire partie, il fallait ou bien en faire déjà partie, ou être membre d'une communauté pour y être élu. Au contraire, d'André mit en place le principe, base de la nouvelle constitution, d'une élection entre citoyens égaux, autrement dit un système où les individus étaient considérés indépendamment de leur appartenance à une communauté.

Cependant, si le premier système revendiqué par les corporations avait pour caractéristique de leur garantir une participation au conseil, ce n'était pas le cas du système nouveau, qui n'offrait aucune garantie à aucun corps organisé de participer au pouvoir municipal. Dans le premier système, ceux qui appartenaient à un corps avaient une garantie d'envoyer un des leurs au conseil. Dans le second système, si tous les citoyens remplissant les critères d'éligibilité avaient une chance formellement égale d'être choisis

(76) Le règlement de convocation se trouve sur une affiche, AMM, 1BB 3335. Pour être électeur, il suffisait d'être chef de famille, avoir plus de vingt-cinq ans et être domicilié à Marseille.

(77) AMM, BB 224 f 217 ; Jean-Louis LAPLANE, op. cit., p.185, donne les qualités des élus. 
au conseil de ville, plus personne n'avait de garantie de pouvoir participer à l'exercice du pouvoir.

On touche là à un enjeu essentiel qui concerne le sens de l'usage de l'élection sur base égalitaire - et non pas simplement de l'élection - comme procédure d'accès aux charges. Pour interpréter la signification de chacun de ces deux systèmes, il est nécessaire de se demander qui avait intérêt à défendre l'un plutôt que l'autre ${ }^{78}$. Si les maîtres avaient intérêt au premier système, qui leur offrait une garantie de participation au pouvoir politique, qui avait intérêt au second ? C'est un phénomène empiriquement attesté qu'en l'absence de candidatures déclarées, l'élection agit nécessairement comme un filtre social, de manière analogue à un cens ${ }^{79}$. C'est tout à fait ce que l'on observe dans le scrutin d'octobre 1789, comme le montre bien son résultat.

Considérer alors l'élection sur base égalitaire comme étant le système le plus légitime reviendrait, en tant qu'historien, à souscrire sans le dire au système dominant, autrement dit à oublier de dire que tous n'avaient pas un intérêt égal à le mettre en place, et qu'il existait des possibles latéraux, des alternatives. La légitimité de l'élection sur base égalitaire n'est en effet rien d'autre que le produit d'une lutte historique gagnée par ceux qui avaient le plus intérêt à cette procédure, une lutte ayant consacré l'aspect formellement égalitaire de l'élection tout en masquant son aspect socialement inégalitaire.

Par ailleurs, dans le droit fil des pratiques corporatives où il était courant d'utiliser le mandat impératif, certains élus dans les quartiers en octobre arrivèrent au conseil de ville munis de tels mandats. Le 10 octobre, d'André, qui présidait le nouveau conseil renforcé des députés des quartiers, dit avoir constaté que certains élus étaient porteurs de mandats

(78) Un intérêt objectif, autrement dit empiriquement constaté, ce qui ne signifie pas qu'il soit connu consciemment par les acteurs eux-mêmes. Deux cas exemplaires de phénomènes sociaux où les acteurs ajustent leurs pratiques sur leurs chances objectives de succès sans en avoir conscience peuvent-être cités : la reproduction sociale par le biais du système scolaire, où les acteurs sociaux ajustent leurs choix scolaires à leurs chances objectives de succès, et l'énonciation orale de certains phonèmes dans des interactions sociales inégalitaires, voir respectivement : Pierre BoURDIEU, La reproduction, Paris, Minuit, 1970 ; William LABOV, Sociolinguistique, Paris, Minuit, 1976.

(79) Sur le caractère aristocratique de l'élection, au sens d'une procédure ayant, en l'absence de candidature déclarée, la caractéristique de produire une sélection des meilleurs, selon des critères propres à chaque société, voir Patrice GUENIFFEY, Le nombre et la raison, Paris, École des hautes études en sciences sociales, 1993, p. 411-415. On prendra soin aussi de ne pas confondre élection et représentation. Le mécanisme représentatif est un outil de légitimation de la délégation du pouvoir, et n'a rien d'aristocratique. Ceux qui sont choisis par élection ne deviennent pas mécaniquement des représentants, cela dépend de l'ensemble des procédures qui régissent la charge pour laquelle ils ont été choisis. 
impératifs et assène : ils «ont été jugés par l'Assemblée nationale », ils sont « cassables », et il les rejette ${ }^{80}$. Le contenu de ces mandats est inconnu. Mais il est remarquable qu' aucune source ne donne trace de protestations. Une explication pourrait être qu'aucune théorie générale du mandat n'a été produite par les acteurs marseillais, théorie qui a été développée à Paris et dont Maurice Genty a rendu compte ${ }^{81}$. Une telle théorie aurait pu servir aux acteurs à engager une lutte sur ce point précis avec d'André, en défendant le bien fondé - de leur point de vue - du mandat impératif. Il est aussi possible de déduire de cette absence de contestation la légitimité des décisions de l'Assemblée nationale ${ }^{82}$. Car l'habitude qu'avaient les maîtres d'utiliser le mandat impératif et d'être attentifs aux dangers de la délégation du pouvoir aurait dû les inciter à protester.

Que pourrait nous apprendre la confrontation des événements de la chronologie marseillaise avec les différents schémas de révolution municipale proposés par Daniel Ligou ${ }^{83}$ ? Ce dernier dégage trois cas généraux : une absence de révolution municipale, quand l'ancienne administration est restée en place, une révolution « incomplète » quand les pouvoirs anciens sont restés en place à côté de pouvoirs nouveaux, et une révolution «complète » où le pouvoir municipal est exercé dans sa majeure partie par des hommes nouveaux. Cette schématisation pose un premier problème : elle est linéaire, et ne permet pas de décrire des projets qui, tout en transférant le pouvoir à des groupes nouveaux, auraient des significations politiques différentes. C'est typiquement le cas de Marseille. Si le projet des corporations avait réussi, le pouvoir municipal serait passé à terme aux députés des corporations. Mais ces dernières ont été concurrencées par un autre projet, qui emporta la partie comme on vient de le voir. Dans les deux cas, les révolutions municipales auraient été « complètes », et auraient pourtant eu des significations différentes. Par ailleurs, cette schématisation ne prend pas en compte l'évolution de l'organisation du pouvoir à l'intérieur des municipalités. Il ne suffit pas que des hommes accèdent à un conseil municipal pour qu'ils puissent y exercer effectivement le pouvoir. Le conseil renforcé du 13 avril à Marseille l'illustre parfaitement : on a vu comment les échevins triaient les décisions du conseil pour ne relayer que

(80) AMM, BB $224 \mathrm{f}^{\circ} 220 \mathrm{r}$

(81) Maurice GENTY, «Pratique et théorie de la démocratie directe : l'exemple des districts parisiens (1789-1790) », $A H R F, \mathrm{n}^{\circ} 259,1985$, p. 8-24.

(82) «Légitimité », au sens d'usage « dominant et méconnu comme tel », qui « produit ses effets en ayant l'air de ne pas être ce qu'il est », voir Pierre BoURDIEU, Questions de sociologie, Minuit, 1984, p. 95-112.

(83) Daniel Ligou, op. cit. 
celles qu'ils jugeaient utiles, écartant les autres, comme celle demandant la légalisation de l'entrée des corporations à la municipalité.

Outre l'implication des corporations, une étude des révolutions municipales devrait être très attentive aux changements de l'organisation du pouvoir, en documentant l'élargissement de fait et de droit de l'initiative politique dans les conseils municipaux, autrement dit en cherchant à savoir qui pouvait faire des propositions au conseil, si les délibérations étaient imprimées ou même publiques, quelles étaient les techniques délibératives, qui avait la maîtrise de l'ordre du jour, entre autres.

\section{Les députés des corporations au conseil de ville : mars-octobre 1789}

Reprenons l'arc chronologique correspondant à la présence des députés des corporations au conseil de ville pour en suivre la politique. Les députés des corporations participèrent au conseil de ville pendant deux périodes. Du 24 mars au 14 mai, puis du 18 juillet au 14 septembre. Dans la première période, de grandes décisions sont prises : l'abolition du régime des impôts, la nomination de 24 commissaires pour créer un impôt qui ne porte pas sur les comestibles, un emprunt de 600000 livres pour subvenir aux besoins immédiats de la communauté, et le maintien d'un conseil de ville renforcé entre autres par les députés des corporations. Dans cette période, les députés des corporations d'arts libéraux et mécaniques réunis forment $60 \%$ du conseil. Et les décisions du conseil sont dans le droit fil des cahiers de doléances des corporations. Les échevins gardaient cependant un autre pouvoir important : la maîtrise de la correspondance officielle. Par exemple, suite au conseil du 13 avril où il fut délibéré de demander au roi de sanctionner légalement l'élargissement du conseil de ville, les échevins n'écrivirent aucune lettre en ce sens, au contraire, ils critiquèrent cette composition nouvelle.

Dans la deuxième période, après le 18 juillet, le conseil fut composé en moyenne à $51 \%$ par les députés des corporations. La participation générale fut en moyenne deux fois moins importante durant cette deuxième période : 86 personnes contre 164 présents dans la première période, en comptant les conseillers de ville ordinaires et les députés des corporations. Le conseil renforcé délibéra notamment sur les propositions d'une assemblée de citoyens réunis dans la taverne d'Arquier le 23 juillet ${ }^{84}$. Ces

(84) Le manuscrit est dans AMM, BB 224, imprimé dans AD BDR, 16 F 34, édité dans Jean-Louis LAPLANE, op. cit., p.226. 
derniers demandaient plusieurs choses, dont la cessation de toute correspondance avec « les gens proscrits », notamment l'intendant, l'enlèvement des troupes logées chez l'habitant, la publicité des séances du conseil de ville, et une réforme de la garde bourgeoise. Concernant cette garde bourgeoise, le conseil de ville délibéra de demander aux officiers de cesser de tenir des assemblées au sein de leur « comité permanent». Mais c'est Caraman qui réforma la garde, en y faisant entrer les corporations ${ }^{85}$. Le conseil délibéra aussi le 3 août que les troupes réglées seraient considérées comme « troupes nationales » et qu'en conséquence le roi devrait défrayer les citoyens qui les hébergeaient. Par ailleurs, rien n'indique que les séances du conseil fussent jamais rendues publiques. Par contre, à partir du 8 octobre, soit après l'éviction des députés des corporations, le conseil fut convoqué par affiches, et ses délibérations généralement imprimées.

Sur un aspect majeur le conseil suivit les propositions de l'assemblée d'Arquier, puisqu'il choisit le 30 juillet de consommer l'opposition contre l'intendant La Tour en cessant toute correspondance avec lui. C'était une décision politique forte, puisque le conseil de ville rompait avec le principal agent du roi en Provence, avec qui les échevins échangeaient une abondante correspondance avant 1789. Par ailleurs, le conseil continua de son propre chef le chemin vers une forme d'imposition nouvelle. Il délibéra le 27 août une contribution provisoire de $10 \%$ du prix de la location des biens immeubles, payable moitié par le locataire moitié par le propriétaire. Il obtint rapidement une homologation par la Cour des comptes, le 31 août, puis délibéra un plan d'application le 7 septembre. Au 19 novembre, cette contribution avait produit environ 300000 livres ${ }^{86}$. Le conseil continua aussi son effort pour éclaircir les comptes de la communauté des années 1787 et 1788.

En somme, le conseil de ville tint une position intermédiaire entre les tenants de l'Ancien Régime et les partisans de réformes plus radicales. En effet il ne réussit ou ne voulut pas réformer la garde bourgeoise pour lui donner une composition plus populaire et surtout des chefs élus, il ne rendit pas ses séances publiques, mais il continua néanmoins le travail de réforme des impôts, de rejet de la concentration du pouvoir dans les mains de l'intendant, d'éclaircissement des comptes de la communauté, et par sa seule existence, le conseil concrétisait deux formes différentes d'ouverture 
de la participation au pouvoir politique, tout d'abord aux députés des corps de métier, puis à ceux des quartiers.

\section{Diffusion de la politisation dans l'espace des corporations}

Au terme de l'année 1789 dont on vient de dérouler la chronologie, il est légitime de s'interroger sur un éventuel rapport entre l'hétérogénéité socio-économique du monde corporatif et le contenu des revendications corporatives. En l'absence de toute source fiscale, il est impossible d'établir une classification précise des corporations en termes de richesse de leurs membres. Concernant le capital culturel, le seul critère quantifiable, est le taux de signatures ${ }^{87}$. Par ailleurs, on peut obtenir pour chaque corporation un indicateur de son engagement en comptant le nombre d'assemblées ayant pour enjeu des objets politiques non strictement corporatifs. Or il n'y a aucune corrélation entre le taux de signatures et le nombre de délibérations politiques, pas plus qu'avec le fait d'être une communauté d'art libéral ou mécanique. Concernant le contenu des revendications portées dans les doléances en mars, le fait dominant est leur unanimité, indépendamment de tout critère distinguant les communautés entre elles. Toutes veulent la réforme des impôts, toutes veulent l'agrandissement du conseil de ville et pouvoir y députer directement, et une grande majorité veut l'abolition pure et simple des fermes ${ }^{88}$.

Concernant la participation à la première garde bourgeoise, les corporations sollicitées par les échevins expriment des revendications homogènes : pouvoir élire les officiers, ne pas participer en tant que corps mais en tant que citoyens, autrement dit laisser à chacun sa liberté. Ce refus de servir en tant que corps, fut renouvelé début juin la première fois que Caraman tenta de les faire entrer dans la garde réformée. Ce n'est qu'en septembre que deux groupes distincts se dessinent, avec ceux qui refusent une participation à la garde ou émettent des critiques sur sa formation, autrement dit qui persistent dans leur position initiale, et ceux qui acceptent. Ici encore il n'y a aucune corrélation entre l'acceptation de la participation à la garde avec le taux de signatures ou avec l'intensité de la participation politique.

(87) Le fait de savoir signer étant corrélé avec la connaissance de la lecture, voir François FURET et Jacques OzOUF, Lire et écrire. L'alphabétisation des Français de Calvin à Jules Ferry, Paris, Minuit, 1977.

(88) Seuls les avocats posent des limitations à cette députation. L'abolition des fermes n'est pas demandée par les corps suivants : avocats, chaudronniers, cordonniers, colporteurs, couteliers, fripiers, horlogers, huissiers, jardiniers, liqueristes, magaziniers-saleurs, menuisiers. 
Entre mars et octobre, le conseil renforcé se réunit vingt et une fois. Une moyenne sur toutes les corporations et toutes les séances montre que les députés des corporations d'arts libéraux y sont présents neuf fois sur vingt et une, ceux des arts mécaniques dix fois sur vingt et une, autrement dit, aucune différence significative entre les deux groupes. Aucune corrélation ne peut être trouvée entre le nombre de délibérations politiques d'une communauté et la fréquence moyenne de présence de ses députés au conseil. Autrement dit, ce n'est pas parce qu'une communauté se réunit souvent pour délibérer sur les affaires de la ville que ses députés participent plus souvent que les autres aux séances du conseil renforcé.

De plus, aucune trace de conflits entre corporations autour des enjeux politiques municipaux et nationaux ne peut être trouvée. C'est que l'heure n'était pas aux conflits entre corporations. Ces dernières étaient conscientes de ce risque de division, et c'est entre autres pour cela qu'elles refusèrent au mois d'avril de participer en tant que corporations à la garde de ville, pour ne pas générer des conflits de préséances qu'elles ne connaissaient que trop bien, en ce moment où, au contraire, une victoire dépendait de leur convergence de vues. Le phénomène dominant est donc que la politisation des communautés de métier, dans son contenu et dans sa dynamique, semble indépendante de leur position dans les systèmes de classement d'Ancien Régime, et indépendante de leur hétérogénéité socio-économique.

Par ailleurs, le rapport entre maîtres et ouvriers ne semble pas avoir été bouleversé au début du processus révolutionnaire. Les ouvriers étaient dépossédés de tout moyen légal d'organisation politique, et les maîtres ne leur ont jamais ouvert leurs assemblées ${ }^{89}$. En aucun cas les maîtres ne cherchèrent à représenter ou à porter les intérêts de leurs ouvriers. Par ailleurs, à l'intérieur des corporations, les syndics ne semblent pas avoir joué de rôle dominant. Un indice en est que dans $80 \%$ des cas, les députés des corporations ne sont pas syndic ou prieur en exercice, autrement dit que les maîtres n'ont pas donné un pouvoir supplémentaire à ceux qui dirigeaient déjà, pour l'année en cours, les délibérations de leur corps.

Les corporations de métier ont bien été un acteur important des luttes politiques à Marseille en 1789. Pour faire émerger leur rôle réel, il fallait prêter attention aux sources permettant de reconstruire leur activité, leur positionnement politique et l'intensité de la mobilisation des maîtres. Ces sources existent pour de nombreuses autres villes dans les fonds

(89) À l'exception des emballeurs, le 18 mars 1789, pour l'élection aux États généraux, et le 15 avril à propos de la garde bourgeoise, ADBDR, 394 E 84. 
départementaux et municipaux, et mériteraient une nouvelle exploration, dont l'histoire du début de la Révolution française bénéficierait grandement. Les communautés de métier n'étaient pas, dans de grandes parties du royaume, ces groupes en déclin décrit par l'historiographie. Au contraire, les maîtres étaient, parmi tous les acteurs urbains, les seuls à posséder une organisation légale de nature politique.

L'historiographie permet, avec plus ou moins de précision, de faire l'hypothèse que le cas marseillais n'est pas une exception. Mais dans la majorité des études publiées, les archives des communautés ont été laissées de côté, ainsi que les minutes notariales. Il est donc souvent impossible de savoir, en cas d'absence des communautés de métier du tableau des événements, si cette absence est le résultat d'un défaut d'activité politique, ou plutôt un effet des principes de vision des chercheurs ayant exclu a priori les corporations du champ de leurs investigations.

Concernant les conditions nécessaires à l'entrée dans la lutte politique en 1789, dans une ville comme Marseille, la comparaison entre les ouvriers et les maîtres est éclairante ${ }^{90}$. En effet, ce qui semble conditionner les différences de pratiques politiques entre maîtres et ouvriers, en 1789, n'est pas directement leur positionnement relatif dans l'espace social, mais leur inégale maîtrise de moyens organisationnels. Les ouvriers ne disposaient pas de moyens légaux d'organisation politique. Or ces moyens sont bien ici une condition nécessaire à une action prolongée et soutenue.

Les maîtres étaient porteurs d'une forte culture d'assemblée, maîtrisant un ensemble de procédures pratiques telles que l'élection, la délégation du pouvoir, la reddition des comptes, les votes à la majorité pour trancher des décisions. Ainsi, il conviendrait de nuancer le propos de Paula Cossart qui avance que la culture d'assemblée «n'était jusque-là pas celle de la population $»^{91}$. Cette culture n'a pu que faciliter leur appropriation des

(90) Ouvriers au sens des classifications d'Ancien Régime. Par exemple, de ce point de vue, les portefaix ne sont pas considérés simplement comme des ouvriers puisqu'ils formaient un corps. (91) Paula COSSART, « S'assembler en Provence sous la Révolution », $A H R F, \mathrm{n}^{\circ} 331,2003$, p. $57-77$, p.71. 
institutions mises en place par la Révolution, à commencer par l'assemblée de section.

Laurent HENRY Université Paris I Panthéon-Sorbonne / IHRF La Source, 15 Chemin de Bédouffe 13109 Simiane Collongue laurent.louis.henry@gmail.com 